\title{
RGS Domain
}

National Cancer Institute

\section{Source}

National Cancer Institute. RGS Domain. NCI Thesaurus. Code C14107.

Typically 120 amino acids long, the RGS (Regulator of G protein Signaling) Domain is found in over 20 human proteins, bind activated G-alpha subunits, and are responsible for GAP function. Through other domains (e.g., DEP, PDZ, or PH) RGS proteins link to other signaling molecules. RGS domains contain conserved contiguous, or dispersed, GH subdomains (GH1, 2, and 3). All RGS domains share a tertiary structure of nine alpha helixes that form a right-handed anti-parallel bundle of helixes 4-7 and a terminal bundle of helixes 1-3 and 8-9. The 3-4, 5-6, and 7-8 helix loops form an interaction surface between RGS4 and Ga(i). RGS domains allosterically stabilize the G-alpha GTP binding pocket, accelerating GT Pase activity. Thus, RGS Family members rapidly inactivate G protein signal transmission from seven transmembrane receptors in GPCR signaling pathways. $(\mathrm{NCl})$ 